\title{
Definite Integrals of the Complete Elliptic Integral $K^{*}$
}

\section{L. Glasser**}

\section{(January 26, 1976)}

\begin{abstract}
Definite integrals whose integrands contain the complete elliptic integral of the first kind are compiled.
\end{abstract}

Key words: Complete elliptic integral; cubic lattice; definite integral; special functions.

\section{INTRODUCTION}

In studying a wide variety of problems involving three dimensional lattices, one is led to examine integrals of the form

$$
\iiint_{0}^{2 \pi} F(\cos x x, \cos m y, \cos n z) d x d y d z
$$

In many cases two of the integrations can be carried out explicitly yielding an integral of the form

$$
\int_{\alpha}^{\beta} F(u) \mathbf{K}(u) d u
$$

where

$$
\mathbf{K}(u)=\int_{0}^{\pi / 2}\left[1-u^{2} \sin ^{2} \theta\right]^{-1 / 2} d \theta
$$

is the complete elliptic integral of the first kind. Examples of this are contained in references [1] ${ }^{1}$ and [2]. In the studying of the vibrational properties of a harmonically coupled simple cubic lattice of mass points, the author was led to evaluate a large number of these integrals. 
The literature concerning integrals of the form (2) is rather sparse. The known results, due mainly to Kaplan [3] and Byrd and Friedman [4] are summarized by Gradshteyn and Ryzhik [5] as entries 6.141-6.147 and 5.153. For completeness these older results have been included in the following table. The methods by which these formulas have been obtained are diverse and it would be impractical to go into the derivations in detail. Several, such as (1-18) were obtained quite simply by integrating the series representing $\mathbf{K}(u)$ term by term. Others, e.g., (II-10) were found by applying the Fourier inversion theorem to a known integral. Several results were obtained by writing $\mathbf{K}(u)$ as a hypergeometric function and applying the general formulas of section 7.5 of reference [5]. Occasionally more complicated procedures were needed (e.g., see [6]). When possible the results were independently checked by considering special cases.

It is felt that the publication of a table of these formulas would be useful to others working in this field as well as form a nucleus for a more extensive tabulation by others in the future. In addition, a number of our results have a certain intrinsic charm.

The notation used in the following tables is that of the Bateman manuscript project series [7]. In addition, $\theta(\mathrm{u})$ denotes the unit step function which is unity when $u>0$ and vanishes for $u<0$. The conditions on the parameters for which the formulas are valid, when not explicitly noted, are easily ascertained and are generally those under which both sides are defined. Finally, these table partially supplement the recent tabulation of representations of the complete elliptic integral as Bessel function integrals by Okui [8].

I. Algebraic integrands

\begin{tabular}{|c|c|c|}
\hline & $f(u)$ & $\int_{0}^{1} f(u) \mathbf{K}(u) d u$ \\
\hline & $u^{s-1}\left(1-u^{2}\right)^{(1 / 2)-s}$ & $\frac{\pi^{1 / 2}}{8} \frac{\mathrm{\Gamma}\left(\frac{1}{4}\right)}{\mathrm{\Gamma}\left(\frac{3}{4}\right)} \frac{\mathrm{\Gamma}\left(\frac{1}{2} s\right)}{\mathrm{\Gamma}\left(1-\frac{1}{2} s\right)} \frac{\mathrm{\Gamma}\left(\frac{3}{2}-\mathrm{s}\right) \mathrm{\Gamma}\left(\frac{3}{4}-\frac{1}{2} s\right)}{\mathrm{\Gamma}\left(\frac{5}{4}-\frac{1}{2} s\right)\left(0<\operatorname{Re} s<\frac{3}{2}\right)}$ \\
\hline & $u^{s-1}\left(1-u^{2}\right)^{(1 / 2) s-1}$ & $\frac{2^{-s} \pi^{2}}{2} \frac{\Gamma^{2}\left(\frac{1}{2} s\right)}{\Gamma^{2}\left(\frac{3}{4}\right) \Gamma^{22}\left(\frac{1}{2} s+\frac{1}{4}\right)} \quad(0<\operatorname{Re} s)$ \\
\hline & $u^{s-1}\left(1-u^{2}\right)^{-s / 2}$ & $\begin{aligned} \frac{\pi^{5 / 2}}{4} \csc \left(\frac{1}{2} \pi s\right) \frac{\mathrm{\Gamma}\left(\frac{3}{4}+\frac{1}{4} s\right) \mathrm{\Gamma}\left(\frac{3}{4}-\frac{1}{4} s\right)}{\mathrm{I}^{2}\left(\frac{3}{4}\right) \Gamma\left(\frac{1}{2}+\frac{1}{4}\right) \mathrm{\Gamma}\left(1-\frac{1}{4} s\right)} & (0<\operatorname{Re} s<2)(1-18)\end{aligned}$ \\
\hline & $u^{s-1}\left(1-u^{2}\right)^{-(s+1) / 4}$ & $\frac{\pi^{3 / 2}}{4} \frac{\Gamma\left(\frac{1}{2} s\right) \Gamma^{2}\left(\frac{3}{4}-\frac{1}{4} s\right)}{\Gamma^{2}\left(\frac{3}{4}\right) \Gamma\left(\frac{1}{4} s+\frac{1}{2}\right) \Gamma\left(1-\frac{1}{4} s\right)} \underset{(0<\operatorname{Re} s<3)(1-18)}{(0)}$ \\
\hline (5) & $u\left(u^{2}+z^{2}\right)^{-1}+\left(1+u^{2} z^{2}\right)^{-1}$ & $\frac{\pi}{2}\left(1+z^{2}\right)^{-1 / 2} \mathbf{K}\left[\left(1+z^{2}\right)^{-1 / 2}\right]$ \\
\hline (6) & $\theta(u-z)\left(1-u^{2}\right)^{-1 / 2}\left(u^{2}-z^{2}\right)^{-1 / 2}$ & $\begin{array}{c}2(1+z)^{-1} \mathbf{K}\left[\left(\frac{1-z}{1+z}\right)^{1 / 2}\right] \mathbf{K}\left[\left(\frac{2 z}{1+z}\right)^{1 / 2}\right] \\
(0<z<1)\end{array}$ \\
\hline
\end{tabular}




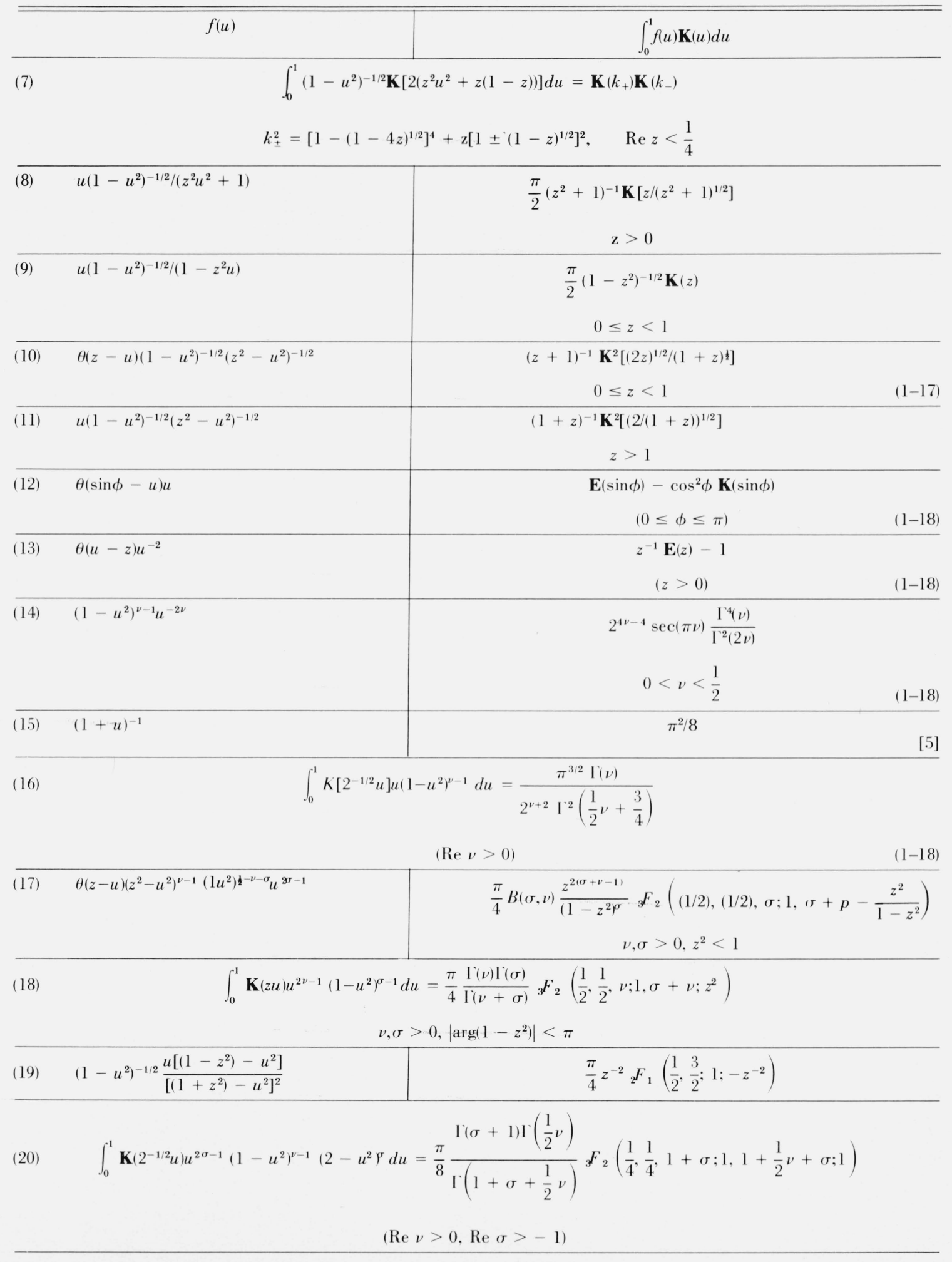




\section{Algebraic integrands-Continued}

\begin{tabular}{|c|c|}
\hline$f(u)$ & $\int_{0}^{1} f(u) \mathbf{K}(u) d u$ \\
\hline$u\left(1-u^{2}\right)^{\nu-1}\left(1-z u^{2}\right)^{-\sigma}$ & $\begin{array}{c}\frac{\pi}{4 \Gamma^{2}\left(\nu+\frac{1}{2}\right)} \quad(1-z)^{\sigma}{ }_{3} F_{2}\left(\nu, \nu, \sigma ; \nu+\frac{1}{2}, \nu+\frac{1}{2} ; \frac{z}{z-1}\right) \\
\nu>0,|\arg (1-z)|<\pi\end{array}$ \\
\hline $\begin{array}{r}\int_{0}^{1}\left(1-u^{2}\right)^{-1 / 2} \mathbf{K}(a u) d u \\
\operatorname{Re}\end{array}$ & $\begin{array}{l}=\frac{\pi^{2}}{4}\left\{{ }_{2} F_{1}\left(\frac{1}{4}, \frac{1}{4} ; 1 ; a^{2}\right)\right\}^{2} \\
a \geq 0\end{array}$ \\
\hline$\theta(z-u) u\left(z^{2}-u^{2}\right)^{\nu-1}$ & $\begin{array}{c}\frac{\pi}{4} \Gamma(\nu) z^{\nu}\left(1-z^{2}\right)^{\nu / 2} P_{-(1 / 2)}^{-\nu}\left(1-2 z^{2}\right) \\
0<z<1, \quad \nu>0\end{array}$ \\
\hline (24) $\quad \theta(u-z) u\left(1-u^{2}\right)^{\nu}\left(u^{2}-z^{2}\right)^{-\nu-(3 / 2)}$ & $\begin{array}{l}\frac{2^{\nu-(3 / 2)}}{\left(1-z^{2}\right)^{\nu+(3 / 2)}} \frac{\Gamma^{2}(\nu+1) \Gamma\left(-\frac{1}{2}-\nu\right)}{\left(c^{2}-1\right)^{2+1) / 4}} P_{-1 / 2}^{-\nu-(1 / 2)}(c) \\
c=(1+z)^{2} /\left(1-z^{2}\right), 0 \leq z<1,-1<\operatorname{Re} \nu<-\frac{1}{2}\end{array}$ \\
\hline$\theta(z-u) u\left(z^{2}-u^{2}\right)^{\nu-1} /\left(1-u^{2}\right)^{\nu+(1 / 2)}$ & $\begin{array}{c}\frac{\pi}{4} I^{\prime}(\nu) z^{\nu}\left(1-z^{2}\right)^{-1} P_{-1 / 2}^{-\nu}\left[\left(1+z^{2}\right) /\left(1-z^{2}\right)\right] \\
\nu>0, \quad 0<z<1\end{array}$ \\
\hline $\begin{array}{c}u^{-1}\left(1-u^{2}\right)^{-1 / 2}\left[1+\frac{z}{\left(z^{2}+x^{2}\right)^{1 / 2}}\right] \\
x^{2}=\left(1-u^{2}\right) / u^{2}\end{array}$ & $\begin{array}{c}(C / 2)^{1 / 2} \frac{4}{\pi}\left[\left(\frac{2}{\xi+1}\right)\left(\xi-\sqrt{\xi^{2}-1}\right)\right]^{1 / 2} \\
\mathbf{K}\left(\sqrt{\frac{\xi-1}{\xi+1}}\right) \mathbf{K}\left(\xi-\sqrt{\xi^{2}-1}\right), \xi=\sqrt{C z} \\
z>1, C=2\left[z-\left(z^{2}-1\right)^{1 / 2}\right]\end{array}$ \\
\hline$\theta(a-u)\left(a^{2} u^{-2}-1\right)^{\nu-1} u^{-2}$ & $\begin{array}{c}\frac{1}{4} e^{\nu \pi i}\left(c^{2}-1\right)^{\nu / 2}(c+1)^{1-v}()_{-(1 / 2)}^{-v}(c) \\
c^{2}=2 a^{-2}-1,0<a<1,0<\operatorname{Re} \nu<\frac{1}{2}\end{array}$ \\
\hline (28) $\quad \theta\left[\left(1+z^{2}\right)^{-1 / 2}-u\right]\left[u\left(1-u^{2}\right)\right]^{-1 / 2}\left[\left(1-u^{2}\right)^{1 / 2}-z u\right]^{-1 / 2}$ & $\begin{array}{c}\frac{1}{2} z^{-1 / 2}\left[Q_{-3 / 4}\left(z+z^{-1}\right) P_{-3 / 4}\left(z+z^{-1}\right)\right. \\
\left.-Q_{-1 / 4}\left(z+z^{-1}\right) P_{-1 / 4}\left(z+z^{-1}\right)\right] \\
(z>0)\end{array}$ \\
\hline (29) $\theta(z-u)\left(1-u^{2}\right)^{-3 / 4}\left[\frac{z\left(1-u^{2}\right)+u\left(1-z^{2}\right)}{z^{2}-u^{2}}\right]^{1 / 2}$ & $\begin{array}{c}\left(\frac{1-z^{2}}{z^{2}}\right)^{1 / 4}\left\{\left[Q_{-1 / 4}(C)\right]^{2}+\left[Q_{-3 / 4}(C)\right]^{2}\right\} \\
0<z<1, C^{-1}=z\left(1-z^{2}\right)^{1 / 2}\end{array}$ \\
\hline (30) $\theta\left[\left(1+z^{2}\right)^{-(1 / 2)}-u\right] u^{2}\left[\frac{u^{2}}{\left(1-u^{2}\right)-z^{2} u^{2}}\right]^{\nu+(3 / 2)}$ & $\begin{array}{l}\frac{2^{(6 \nu+11) / 4}}{|z|^{\nu+1 / 2}} e^{-(\nu+1 / 2 \pi \mathrm{i}} Q_{-(1 / 2)}^{\nu+(1 / 2)}\left(2 z^{2}+1\right) \\
\quad z \text { real, }-3<\operatorname{Re} \nu<-\frac{1}{2}\end{array}$ \\
\hline $\begin{array}{c}\left(1-u^{2}\right)^{\nu-1}\left\{u+z\left(1-u^{2}\right)^{1 / 2}\right]^{-2 \nu} \\
\left.+\left|u-z\left(1-u^{2}\right)^{1 / 2}\right|^{-2 \nu}\right\}\end{array}$ & $\begin{array}{l}2^{4 \nu-3} \frac{\Gamma^{4}(\nu)}{\Gamma^{2}(2 \nu)} \sec (\pi \nu) \times \\
\times{ }_{3} F_{2}\left(\nu, \nu, \nu ; \nu+1 / 2,1 / 2 ;-z^{2}\right) \\
\left(0<z<1,|\nu|<\frac{1}{2}\right)\end{array}$ \\
\hline
\end{tabular}




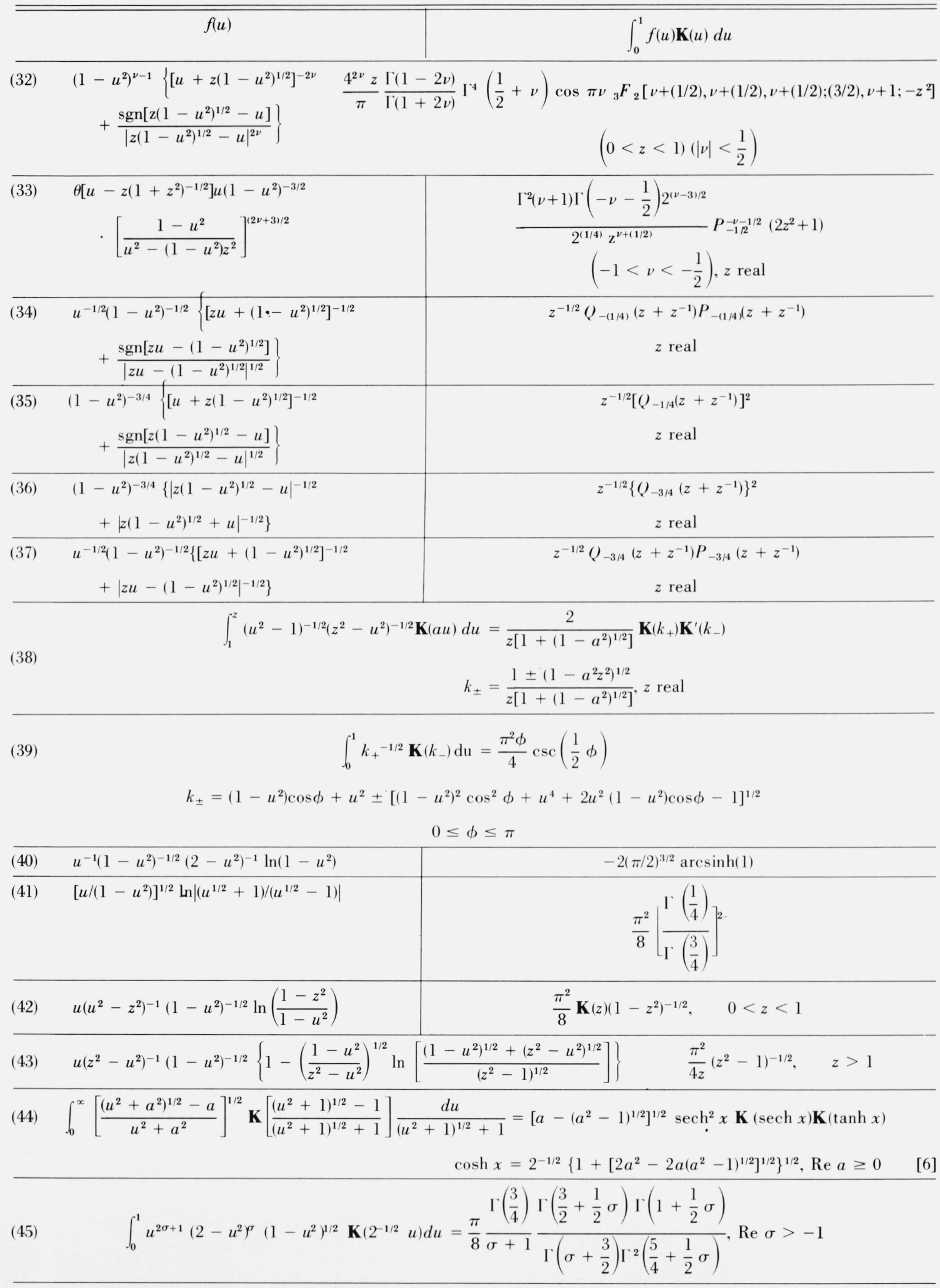


I. Algebraic integrands-Continued

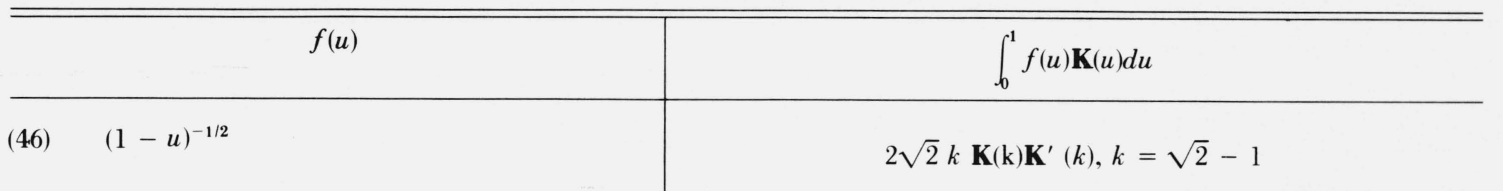

SIAM REVIEW 14, 497 (1972)

(47)

(48)

$$
\int_{z}^{x} u^{-2}\left(u^{-2}-z^{-2}\right)^{a-1} \mathbf{K}(u) d u=\frac{1}{2} \Gamma(a) e^{\pi \mathrm{ia}} z^{-2 a}\left(1-z^{2}\right)^{a / 2} Q_{-1 / 2}^{-a}\left(2 z^{-2}-1\right)
$$

$$
0<a<\frac{1}{2}, \quad 0<z<1
$$

(49) $\int_{\beta}^{1} \mathbf{K}[(1-\beta) u / \beta]\left[(\beta+1) u-\left(u^{2}+\beta\right)\right]^{-1 / 2} d u=\frac{4}{(1+\beta)^{-}}\left\{\frac{(z+1)(z+4) \beta}{(z / 2)^{2}+2 z+2+(2+z)(z+1)^{1 / 2}}\right\}^{1 / 2} \mathbf{K}\left(k_{+}\right) \mathbf{K}\left(k_{-}\right)$

where

$$
\begin{gathered}
z=\frac{2\left[1-\beta+\beta^{2}\right]-2\left[\beta\left(5 \beta-2 \beta^{2}-2\right)\right]^{1 / 2}}{\left[4 \beta-1-\beta^{2}\right]+2\left[\beta\left(5 \beta-2 \beta^{2}-2\right)\right]^{1 / 2}} \\
k_{ \pm}=\left\{\frac{z(\sqrt{z+1} \pm \sqrt{z+4})-2(1-\sqrt{z+1})}{z(\sqrt{z+1} \pm \sqrt{z+4})+2(1+\sqrt{z+1})}\right\}^{1 / 2}, \quad \frac{1}{2} \leq \beta \leq 1
\end{gathered}
$$

318 


\section{Trigonometric integrands}

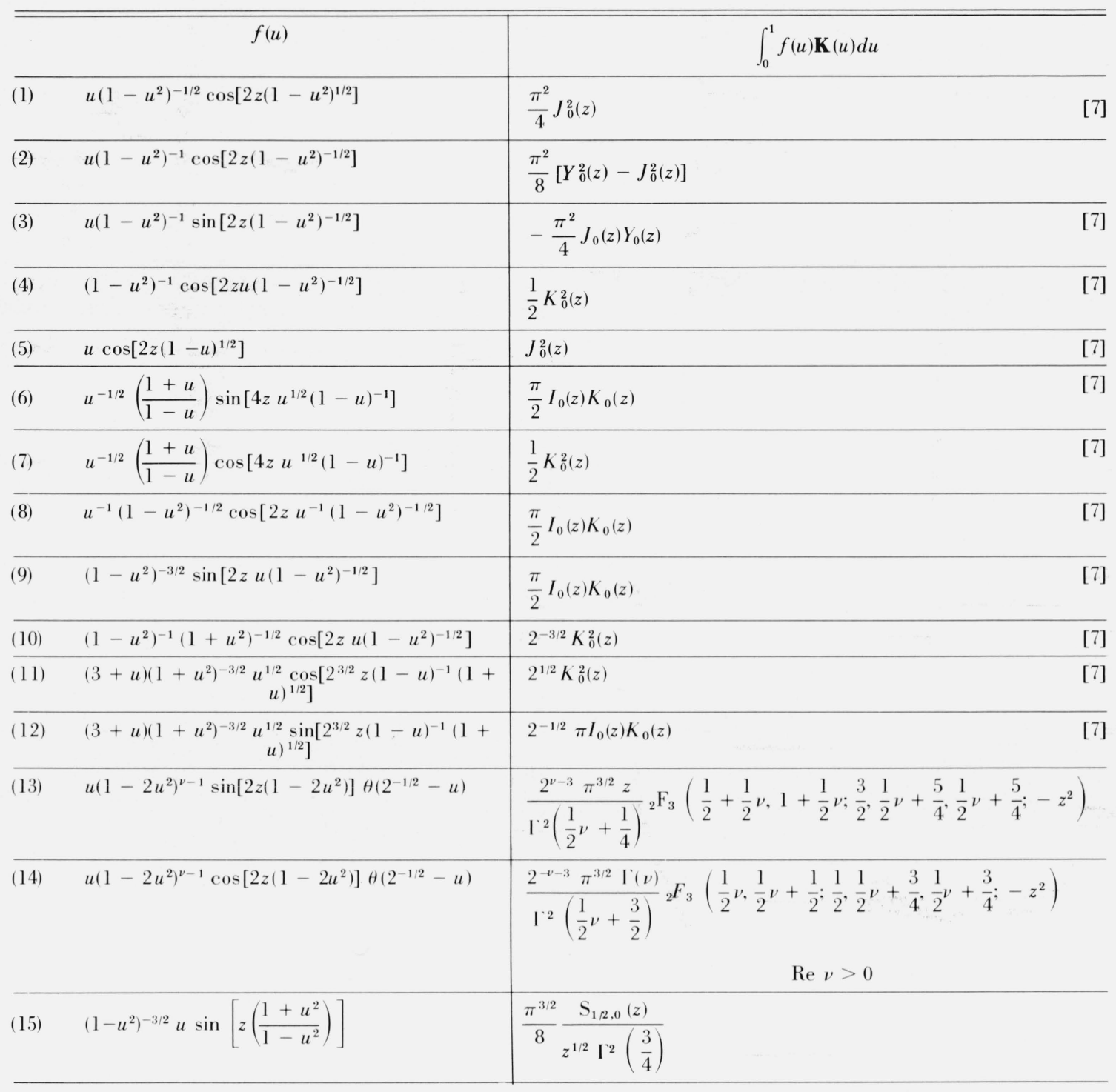


III. Exponential and hyperbolic integrands

\begin{tabular}{|c|c|c|}
\hline \multicolumn{2}{|r|}{$f(u)$} & $\int_{0}^{1} f(u) \mathbf{K}(u) d u$ \\
\hline (1) & $e^{-2 z u}+u^{-1} e^{-2 z / u}$ & $\frac{\pi}{2} I_{d}(z) K_{d}(z)$ \\
\hline (2) & $u\left(1-u^{2}\right)^{-1} \exp \left[-2 z\left(1-u^{2}\right)^{-1 / 2}\right]$ & $\frac{1}{2} K_{0}^{2}(z)$ \\
\hline (3) & $\left(1-u^{2}\right)^{-1} \exp \left[-2 z u\left(1-u^{2}\right)^{-1 / 2}\right]$ & $\frac{\pi^{2}}{8}\left[J_{0}^{2}(z)+Y_{0}^{2}(z)\right]$ \\
\hline (4) & $u\left(1-u^{2}\right)^{-3 / 2} \exp \left[-2 z u^{2} /\left(1-u^{2}\right)\right]$ & $\frac{1}{4}\left(\frac{\pi}{2 z}\right)^{1 / 2} \mathrm{e}^{\mathrm{z}} \mathrm{K}_{0}(\mathrm{z})$ \\
\hline (5) & $u\left(1-u^{2}\right)^{-1 / 2} \exp \left(z u^{2}\right)$ & $\frac{\pi^{2}}{4} e^{z}{ }_{2} F_{2}[(1 / 2),(1 / 2) ; 1,1 ;-z]$ \\
\hline (6) & $u\left(1-u^{2}\right)^{-3 / 2} \exp \left[-4 z u^{2} /\left(1-u^{2}\right)^{2}\right]$ & $\frac{\pi}{16} z^{-3 / 4} e^{\frac{1}{z} z} W_{-1 / 4,0}(z)$ \\
\hline (7) & $u\left(1-u^{2}\right)^{-1}\left(x^{2}-y^{2} u^{2}\right)^{-1 / 2} \exp \left[-\left(\frac{x^{2}-y^{2} u^{2}}{1-u^{2}}\right)\right]$ & $\frac{1}{4}\left(x^{2}-y^{2}\right)^{-1 / 2} K_{0}(x+y) K_{0}(x-y)$ \\
\hline (8) & $\begin{array}{l}u\left(1-u^{2}\right)^{-3 / 2} A^{-1} \exp (-A) \\
\quad A=\left[x^{2}+y^{2}+2 x y\left(\frac{1+u^{2}}{1-u^{2}}\right)\right]^{1 / 2}\end{array}$ & $\frac{1}{4}(x y)^{-1 / 2} K_{0}(x) K_{0}(y)$ \\
\hline (9) & $u^{-2 \nu}\left(1-u^{2}\right)^{\nu-1} \exp \left(-z / 2 u^{2}\right)$ & 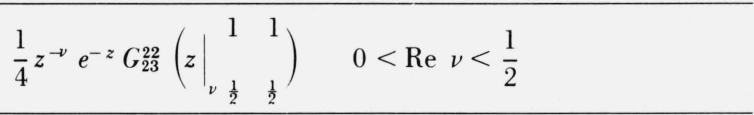 \\
\hline & $u^{2 \nu-1}\left(1-u^{2}\right)^{-v-(1 / 2)} \exp \left[-\frac{1}{2} z\left(\frac{1+u^{2}}{1-u^{2}}\right)\right]$ & 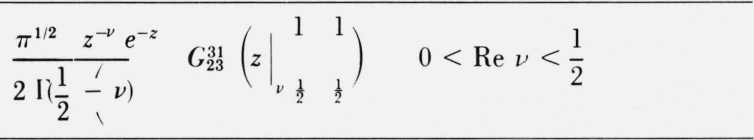 \\
\hline (11) & $\begin{array}{c}u^{\nu+1}\left(1-2 u^{2}\right)^{\nu-1}\left(1-u^{2}\right)^{-(2 v+3) / 4} \exp [-z(1-2 u \\
\cdot \theta\left(2^{-1 / 2}-u\right)\end{array}$ & $\begin{array}{r}\left.\left.1-u^{2}\right)^{1 / 2}\right] \cdot \frac{2^{\nu-(9 / 4)}}{\pi^{1 / 2}} G_{24}^{32}\left(z^{2} \mid \begin{array}{c}1-(\nu / 2) \frac{1}{2}-(\nu / 2) \\
0 \frac{1}{2}(1 / 4)-(\nu / 2)(1 / 4)-(\nu / 2)\end{array}\right) \\
\operatorname{Re} \nu>0\end{array}$ \\
\hline (12) & $u\left(1-u^{2}\right)^{-1 / 2} \cosh \left[2 z\left(1-u^{2}\right)^{1 / 2}\right]$ & $\frac{1}{4} \pi^{2} I_{0}^{2}(z)$ \\
\hline (13) & $\int_{0}^{\infty} \frac{\cos (z u)}{\cosh \left(\left(\frac{1}{2}\right) u\right)} \mathbf{K}(\mathrm{t}$ & $u) d u=\frac{1}{8 \pi}\left|\Gamma\left(\frac{1}{4}+\frac{1}{2} i z\right)\right|^{4}$ \\
\hline
\end{tabular}




\section{Bessel and related functions}

\begin{tabular}{|c|c|c|c|}
\hline & $f(u)$ & $\int^{1} f(u) \mathbf{K}(u) d u$ & \\
\hline$\overline{(1)}$ & $\theta\left(2^{-1 / 2}-u\right) u\left(1-2 u^{2}\right)^{-5 / 2} J_{0}\left[2 z u\left(1-u^{2}\right)^{1 / 2} /\left(1-2 u^{2}\right)\right.$ & $\frac{1}{2} \pi^{1 / 2} z^{-3 / 2} e^{-z}$ & $\operatorname{Re} z>0$ \\
\hline$(2)$ & $u\left(1+u^{2}\right)\left(1-u^{2}\right)^{-5 / 2} J_{0}\left[2 z u /\left(1-u^{2}\right)\right]$ & $2^{-3 / 2} \frac{\pi}{\Gamma^{2}\left(\frac{1}{4}\right)} z^{-3 / 2} K_{0}(z)$ & \\
\hline (3) & $u\left(1-u^{2}\right)^{-3 / 2} J_{0}\left[z u /\left(1-u^{2}\right)^{-1 / 2}\right]$ & $z^{-1} K_{0}(z) \quad \operatorname{Re} z>0$ & [7] \\
\hline (4) & $u\left(1-u^{2}\right)^{-2}\left(1+u^{2}\right)^{1 / 2} J_{0}\left[2 z\left(\frac{1+u^{2}}{1-u^{2}}\right)\right]$ & $-\frac{2^{-1 / 2} \pi}{16 z}\left[\cos (z) Y_{0}(z)+\sin (z) J_{0}(z)\right]$ & $\operatorname{Re} z>0$ \\
\hline (5) & $\theta\left(2^{-1 / 2}-u\right) u\left(1-2 u^{2}\right)^{-1 / 2} J_{0}\left[2 z\left(1-2 u^{2}\right)\right]$ & $\frac{2^{1 / 2} \pi}{16 z} \sin (z) J_{0}(z)$ & $\operatorname{Re} z>0$ \\
\hline (6) & $\int_{0}^{\infty} u^{1 / 2}\left[\frac{2 u}{z^{2}+(u+1)^{2}}\right]^{1 / 2} J_{0}(x t) \mathbf{K}$ & {$\left[\left(\frac{2 u}{z^{2}+(u+1)^{2}}\right)^{1 / 2}\right] d u=\frac{\pi}{x} e^{-z x} J_{0}(x)$} & $x>0$ \\
\hline (7) & $u\left(1+u^{2}\right)^{1 / 2}\left(1-u^{2}\right)^{-2} Y_{0}\left[2 z\left(\frac{1+u^{2}}{1-u^{2}}\right)\right]$ & $\frac{2^{-1 / 2} \pi}{16 z}\left[\cos (z) J_{0}(z)-\sin (z) Y_{0}(z)\right]$ & $\operatorname{Re} z>0$ \\
\hline$(8)$ & $u\left(1-u^{2}\right)^{-3 / 2} K_{0}\left[z\left(1-u^{2}\right)^{-1 / 2}\right]$ & $(\pi / 2 z)^{3 / 2} e^{-z}$ & $\operatorname{Re} z>0$ \\
\hline (9) & $u\left(1+u^{2}\right)^{1 / 2}\left(1-u^{2}\right)^{-2} K_{0}\left[2 z\left(\frac{1+u^{2}}{1-u^{2}}\right)\right]$ & $\frac{2^{-1 / 2} \pi}{16 z} e^{-z} K_{0}(z)$ & $\operatorname{Re} z>0$ \\
\hline$(10)$ & $u\left(1-u^{2}\right)^{-3 / 2} K_{0}\left[z u\left(1-u^{2}\right)^{-1 / 2}\right]$ & $\frac{\pi^{2}}{4 z}\left[\mathbf{H}_{0}(z)-Y_{0}(z)\right]$ & $\operatorname{Re} z>0$ \\
\hline (11) & $u^{2}\left(1-u^{2}\right)^{-2} K_{0}\left[2 z u /\left(1-u^{2}\right)\right]$ & $\frac{2^{-1 / 2} \pi}{8 z^{1 / 2}} S_{-1 / 2,0}(z)$ & $\operatorname{Re} z>0$ \\
\hline (12) & $u^{2}\left(1-u^{2}\right)^{-5 / 2} K_{0}\left[2 z u^{2} /\left(1-u^{2}\right)\right]$ & $\frac{\pi}{16} z^{-3 / 2} S_{1 / 2,0}(z)$ & $\operatorname{Re} z>0$ \\
\hline (13) & $u\left(1-u^{2}\right)^{-3 / 2} \exp \left[-z u^{2} / 2\left(1-u^{2}\right)\right] K_{0}\left[\frac{z}{2\left(1-u^{2}\right)}\right.$ & $\frac{\pi}{2}(\pi / z)^{1 / 2} E_{1}(z)$ & $\operatorname{Re} z>0$ \\
\hline$\overline{(14)}$ & $u\left(1-u^{2}\right)^{-3 / 2} S_{\nu, 0}\left[z\left(1-u^{2}\right)^{-1 / 2}\right]$ & $\frac{\pi}{4 z}\left[\frac{\Gamma\left(-\frac{1}{2} \nu\right)}{\Gamma\left(\frac{1}{2}-\frac{1}{2} \nu\right)}\right]^{2} S_{\nu+1,0}(z)$ & $\operatorname{Re} z>0$ \\
\hline (15) & $u\left(1+u^{2}\right)^{1 / 2}\left(1-u^{2}\right)^{-2} S_{\nu, 1 / 2}\left[z\left(\frac{1+u^{2}}{1-u^{2}}\right)\right]$ & $(\pi / 2)^{1 / 2} 2^{-\nu} \frac{\Gamma^{22}\left(-\frac{1}{2} \nu\right)}{16 z \Gamma\left(\frac{1}{2}-\nu\right)} s_{\nu+1,0}(z)$ & $\operatorname{Re} z>0$ \\
\hline$(16)$ & $u\left(1-u^{2}\right)^{-5 / 4} S_{-1 / 2,1 / 2}\left[2 z\left(1-u^{2}\right)^{-1 / 2}\right]$ & $\frac{2^{-1 / 2} \pi^{3}}{16} z^{-1 / 2}\left[J_{0}^{2}(z)+Y_{0}^{2}(z)\right]$ & $\operatorname{Re} z>0$ \\
\hline$(17)$ & $u^{-2} \mathbf{H}_{0}\left[z u^{-1}\left(1-u^{2}\right)^{1 / 2}\right]$ & $-z K_{0}(z)$ & $\operatorname{Re} z>0$ \\
\hline (18) & $\theta\left(u-2^{-1 / 2}\right) u\left(2 u^{2}-1\right)^{-5 / 2} \mathbf{H}_{0}\left[2 u z\left(1-u^{2}\right)^{1 / 2} /\left(2 u^{2}-1\right.\right.$ & 1)] $-\frac{\pi^{1 / 2}}{8} z^{-3 / 2} e^{-z}$ & $\operatorname{Re} z>0$ \\
\hline
\end{tabular}




\section{Miscellaneous integrands}

\begin{tabular}{|c|c|c|}
\hline & $f(u)$ & $\int_{0}^{1} f(u) \mathbf{K}(u) d u$ \\
\hline (1) & $u\left(1-u^{2}\right)^{-3 / 2} \exp \left[z^{2}\left(\frac{1+u^{2}}{1-u^{2}}\right)^{2}\right] \operatorname{Erfc}\left[z\left(\frac{1+u^{2}}{1-u^{2}}\right)\right]$ & $\begin{array}{c}\frac{1}{16} \Gamma^{2}\left(\frac{1}{4}\right) z^{-3 / 2} e^{(11 / 2) z^{2}} W_{1 / 4,0}\left(z^{2}\right) \\
z \neq 0\end{array}$ \\
\hline (2) & $\begin{array}{l}u\left\{\exp \left[-2 z\left(1-u^{2}\right)^{1 / 2}\right] E i\left[-2 z\left(1-u^{2}\right)^{1 / 2}\right]\right. \\
\left.\quad+\exp \left[-2 z\left(1-u^{2}\right)^{1 / 2}\right] \bar{E} i\left[2 z\left(1-u^{2}\right)^{1 / 2}\right]\right\}\end{array}$ & $-\pi I_{0}(z) K_{0}(z)$ \\
\hline (3) & $u\left(1-u^{2}\right)^{\nu-(1 / 2)} P_{\nu}\left(1-2 u^{2}\right)$ & $\begin{array}{c}\frac{1}{4} \frac{\Gamma^{4}\left(\nu+\frac{1}{2}\right)}{\Gamma^{2}(\nu+1) \Gamma(2 \nu+1)} \\
\nu>-\frac{1}{2}\end{array}$ \\
\hline (4) & $\theta\left(2^{-1 / 2}-u\right) u P_{\nu}\left(1-2 u^{2}\right)$ & $\begin{array}{l}\frac{2^{-1 / 2}}{4\left(\nu+\frac{1}{2}\right)^{2}}\left[\frac{\Gamma\left(\frac{1}{4}\right)}{\Gamma\left(\frac{3}{4}\right)} \frac{\Gamma\left(1+\frac{1}{2} \nu\right)}{\Gamma\left(\frac{1}{2}+\frac{1}{2} \nu\right)} \sin \left(\frac{1}{2} \pi \nu\right)\right. \\
\left.+\frac{\Gamma\left(\frac{3}{4}\right)}{\Gamma\left(\frac{1}{4}\right)} \frac{\Gamma\left(\frac{1}{2}+\frac{1}{2} \nu\right)}{\Gamma\left(1+\frac{1}{2} \nu\right)} \cos \left(\frac{1}{2} \pi \nu\right)\right] \quad \operatorname{Re} \nu>-\frac{1}{2}\end{array}$ \\
\hline (5) & $u P_{\nu}\left(1-2 u^{2}\right)$ & $\begin{array}{r}\frac{\pi \cos (\pi \nu)+2 \sin (\pi \nu)[\gamma+2 \ln 2+\psi(\nu+1)]}{\pi(2 \nu+1)^{2}} \\
\operatorname{Re} \nu>-\frac{1}{2}\end{array}$ \\
\hline (6) & $u\left(1-u^{2}\right)^{-3 / 2} Q_{\nu}\left(\frac{1+u^{2}}{1-u^{2}}\right)$ & $\frac{\pi}{8}\left(\nu+\frac{1}{2}\right)^{2}, \quad$ Re $\nu>-\frac{1}{2}$ \\
\hline (7) & $u^{-2} Q_{\nu}\left(2 u^{-2}-1\right)$ & $\frac{\psi(\nu+1)-\psi\left(\frac{1}{2}\right)}{(2 \nu+1)^{2}}$ \\
\hline (8) & $Q_{v}\left(2 u^{2}-1\right)$ & $\begin{array}{c}\frac{(\pi / 2) \cos (\pi \nu)-[\gamma+2 \ln 2+\psi(\nu+1)]}{(2 \nu+1)^{2}} \\
\nu \neq-\frac{1}{2},-1,-2, \ldots\end{array}$ \\
\hline (9) & $u^{-1}\left(1-u^{2}\right)^{-1 / 2} F_{1}\left[1 ; \frac{1}{2} ;-2 z\left(1-u^{2}\right) / u^{2}\right]$ & $\frac{\pi}{4} e^{z} K_{0}(z)$ \\
\hline (10) & $u\left(1-u^{2}\right)^{-1 / 2}{ }_{1} F_{1}\left(\frac{3}{2} ; \frac{1}{2} ;-2 z\left(1-u^{2}\right)\right)$ & $\frac{\pi^{2}}{4} I_{0}(z) e^{-z}$ \\
\hline (11) & $u^{-1}\left(1-u^{2}\right)^{-1 / 2}{ }_{2} F_{1}\left(\frac{1}{2} \nu+\frac{1}{4}, \frac{1}{4}-\frac{1}{2} \nu ; \frac{1}{2} ; 1-u^{-2}\right)$ & $\frac{\pi}{2} \frac{\Gamma\left(\frac{1}{4}+\frac{1}{2} \nu\right)}{\Gamma\left(\frac{3}{4}+\frac{1}{2} \nu\right)} \frac{\Gamma\left(\frac{1}{4}-\frac{1}{2} \nu\right)}{\Gamma\left(\frac{3}{4}-\frac{1}{2} \nu\right)}$ \\
\hline (12) & $\int_{0}^{1}(1-u)^{\nu-1}(1-u z)^{-\sigma} \mathbf{K}\left[(u z)^{/ 2} \mathfrak{b} F_{1}\left[\sigma,-\frac{1}{2} ; \nu\right.\right.$ & $\left.\frac{(1-u) z}{1-u z}\right] d u=\frac{\pi}{2 \nu_{2}} F_{1}\left(\sigma+\frac{1}{2}, \frac{1}{2} ; \nu+1 ; \mathrm{z}\right)_{\operatorname{Re} \nu>0}$ \\
\hline (13) & $\int_{0}^{1}(1-u)^{\nu-2} K\left[(u z)^{1 / 2}\right]_{2} F_{1}\left[\nu-\frac{1}{2}, \nu-\frac{1}{2} ; \nu-1 ; x(1-u)\right.$ & $\begin{array}{r}d u=\frac{\pi}{2(\nu-1)}(1-x)^{1-\nu}{ }_{2} F_{1}\left(\frac{1}{2}, \frac{1}{2} ; \nu ; z+x-z x\right) \\
\operatorname{Re} \nu>1\end{array}$ \\
\hline
\end{tabular}


A formula which was obtained as a special case of another in the table is indicated by a reference in parentheses. For example, (II-7) refers to formula (7) of section II dealing with trigonometric integrals. When possible a result related to a published one is indicated by a reference in square brackets.

The author will be grateful for being informed of any errors found in the table, or of additional results.

\section{References}

[1] Watson, G. N., Three triple integrals, Q. J. Math. Oxford 10, 266 (1939).

[2] Morita, T., and Horiguchi, T., Formulas for the lattice Green's functions for the cubic lattices in terms of the complete elliptic integral, J. Phys. Soc. (Japan) 30, 957 (1971).

[3] Kaplan, E. L., Multiple elliptic integrals, J. Math. and Physics 29, 69-75 (1950).

[4] Byrd, P. F., and Friedman, M. D., Handbook of Elliptic Integrals for Engineers and Physicists (Springer Verlag, Berlin 1954).

[5] Gradshteyn, I. S., and Ryzhik, I. M., Tables of Integrals Series and Products (Academic Press, 1965).

[6] Glasser, M. L., An elliptic integral identity, Math. Comp. 25, 533 (1971). That the restriction on a is that given in 144, rather than the one in this paper was pointed out by H. Fettis (private communication).

[7] Erdélyi, A., Magnus, W., Oberhettinger, F., and Tricomi, F. G., Tables of Integral Transforms, Vols. 1,2 (McGrawHill Book Co., Inc., New York, 1954).

[8] Okui, S., Complete elliptic integrals resulting from infinite integrals of Bessel functions. Nat. Bur. Stand. (U.S.), J. Res. 78B (Math. Sci. No. 3, 113-134 (July-Sept. 1974).

(Paper 80B2-446) 